\title{
Terapia de Aceptación y Compromiso en el tratamiento del Trastorno Obsesivo-Compulsivo.
}

Acceptance and Commitment Therapy in the treatment of Obsessive-Compulsive Disorder.

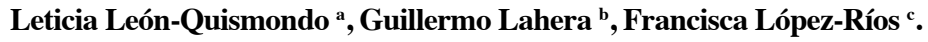 \\ ${ }^{a}$ Servicio de Psiquiatría. Hospital Universitario Príncipe de Asturias, España ${ }^{b}$ Departamento de Medicina \\ y Especialidades Médicas. Universidad de Alcalá, España. ${ }^{c}$ Departamento de Psicología. Universidad de \\ Almería, España.
}

Correspondencia: Guillermo Lahera Forteza (guillermo.lahera@uah.es)

Recibido: 28/01/2014; aceptado: 15/06/2014

\begin{abstract}
RESUMEN: El tratamiento psicológico de elección del Trastorno Obsesivo-Compulsivo (TOC) es la Exposición con Prevención de Respuesta (EPR). El objetivo de este trabajo es llevar a cabo un análisis funcional de los procedimientos de EPR y Terapia de Aceptación y Compromiso (ACT) en el tratamiento del TOC, y comparar la eficacia de ambas formas de terapia. Para ello, se realizó una revisión sistemática de la literatura disponible hasta el momento. Los resultados obtenidos indican que estas formas de intervención se basan en diferentes procesos de cambio, pudiendo constituir la ACT una buena alternativa para aquellas personas que no se beneficien de un tratamiento con EPR, además de para lograr una prevención de recaídas en el futuro. PALABRAS CLAVE: Exposición con Prevención de Respuesta, Terapia de Aceptación y Compromiso, Trastorno Obsesivo-Compulsivo.
\end{abstract}

ABSTRACT: The psychological treatment of obsessive-compulsive disorder (OCD) is the exposure with response prevention (ERP). The aim of this work is to perform a functional analysis of the EPR and Acceptance and Commitment Therapy (ACT) procedures in the treatment of OCD, and to compare the efficacy of both forms of intervention. A systematic review of the literature was performed. The results indicate that these interventions are based on different processes of change; the ACT may be a good alternative for those who do not have access to treatment with EPR and in the relapse prevention.

KEY WORDS: Exposure with Response Prevention, Acceptance and Commitment Therapy, Obsessive-Compulsive Disorder.

\section{Introducción}

Los tratamientos que han demostrado su eficacia hasta el momento en el trastorno obsesivo-compulsivo (TOC) son el tratamiento de exposición con prevención de respuesta (EPR) y el tratamiento farmacológico (1). La EPR tiene un impacto clínicamente significativo en la mayoría de los pacientes (2-3) pero, sin embargo, tiene una tasa de deserción del $25 \%$ (2) y una tasa de rechazo de entre el $5 \%$ y el $22 \%$ de los participantes (4-5). Esto puede deberse en parte a que es un tratamiento intensivo y potencialmente desagradable para el paciente, ya que éste debe exponerse a una situación temida y permanecer en esa situación durante un período significativo de tiempo. Este aspecto, junto con la gran comorbilidad que presenta el TOC con los trastornos del estado de ánimo, de ansiedad o por abuso de sustancias (2), hace que el abordaje de este problema sea a veces complejo. 
En este sentido, el surgimiento de la Terapia de Aceptación y Compromiso (ACT) (6), supone una nueva forma de tratamiento para los trastornos de ansiedad, que se basa en "dar a la gente la oportunidad de experimentar ansiedad sin estar luchando con la ansiedad" (7). Esto es lo que la ACT llama "flexibilidad psicológica" (8): la participación en las conductas positivas en lugar de tratar de evitar dificultades, experiencias y emociones. Desde sus inicios en 1987 bajo el nombre de Terapia Contextual (7), hasta el primer pequeño manual (9) y el genérico (6) se ha ido desarrollando y aplicando al tratamiento de numerosos trastornos, como el trastorno de angustia con agorafobia (10-11), el trastorno de ansiedad generalizada (12-13), los síntomas psicóticos (14-15), el abuso de alcohol (16-17), el duelo complicado (18), la depresión (19-20) o el dolor crónico (21-22). Algunos trabajos plantean que la ACT puede resultar una alternativa a la EPR en el tratamiento del TOC. El presente trabajo pretende describir los procedimientos de EPR y ACT en el tratamiento del TOC y comparar la eficacia de ambas intervenciones.

Método.

La presente revisión bibliográfica se basa en estudios que utilizan la exposición con prevención de respuesta y la terapia de aceptación y compromiso para el tratamiento del trastorno obsesivo-compulsivo. Se han utilizado estudios de eficacia, estudios de caso y revisiones sistemáticas. Los criterios principales de inclusión de los estudios fueron los siguientes: 1. Estudios que utilizaran la terapia cognitivo-conductual como tratamiento para el trastorno obsesivo-compulsivo. 2 . Estudios que aplicaran la exposición con prevención de respuesta como tratamiento para el trastorno obsesivo-compulsivo. 3. Estudios que utilizaran la terapia de aceptación y compromiso como tratamiento para el trastorno obsesivo-compulsivo. 4. Estudios que compararan la aplicación de terapia cognitivo conductual o exposición con prevención de respuesta con terapia de aceptación y compromiso para el trastorno obsesivo compulsivo.

Esta revisión fue específica y sistemática, ya que se seleccionaron los estudios que cumplían los criterios anteriores. Se emplearon principalmente las siguientes bases de datos: "PubMed", "PsycINFO", "Cochrane" y "MDConsult". Los estudios seleccionados abarcaron los últimos seis años. Los estudios fueron identificados utilizando dos estrategias de búsqueda. En primer lugar, se aplicaron los siguientes comandos: (cognitive and behavioral therapy OR exposure with response prevention OR acceptance and commitment therapy) AND (obsessive compulsive disorder); (acceptance and commitment therapy) AND (cognitive and behavioral therapy OR exposure with response prevention) AND (obsessive com- 
ORIGINALES Y REVISIONES

pulsive disorder). En segundo lugar, se examinaron las listas de referencias de los artículos que cumplieron los criterios de inclusión para la revisión.

\section{La Exposición con Prevención de Respuesta (EPR)}

La EPR se basa en la teoría del aprendizaje, que postula que las obsesiones evocan ansiedad y angustia, mientras que las compulsiones o rituales reducen dichos síntomas. La EPR se basa en la suposición de que las obsesiones producen una ansiedad y un malestar marcados, mientras que las compulsiones sirven para reducir esta ansiedad. De esta forma, aunque las compulsiones sirven de forma temporal para reducir la ansiedad, las obsesiones permanecen, porque al realizar la compulsión no se desarrolla habituación. Así, el comportamiento compulsivo permanece porque es reforzado por la reducción de la ansiedad. La EPR está dirigida a interrumpir este patrón. Para ello, se expondrá al paciente a situaciones que le producen un malestar obsesivo y se evitará que el paciente lleve a cabo comportamientos compulsivos (23).

Desde la perspectiva de la EPR, la intervención con personas con TOC se basa en tres principios fundamentales: exponer a los pacientes a los estímulos ansiógenos asociados a sus obsesiones; impedir que los pacientes realicen durante las exposiciones conductas de evitación o neutralización observables o no (compulsiones o rituales) encaminados a reducir la ansiedad (prevención de respuesta); y favorecer la gestión de la ansiedad y las emociones asociadas a las obsesiones. El objetivo principal de la EPR es que el paciente pueda adquirir, mediante el aprendizaje de estrategias y técnicas, un sentimiento de control y eficacia, ante la respuesta provocada automáticamente por los pensamientos o imágenes obsesivos (23).

Existen estudios que muestran que la EPR es efectiva en el tratamiento del TOC (23-25). Se ha comparado la utilización de la EPR con otros tratamientos para el TOC, en estudios controlados con asignación aleatoria a las condiciones experimentales, resultando más eficaz la EPR que el entrenamiento en control de la ansiedad (26) o la terapia cognitiva (27).

\section{La Terapia de Aceptación y Compromiso (ACT).}

La ACT no es sólo una propuesta terapéutica, es un modelo terapéutico que incluye una concepción filosófica, una aproximación psicopatológica, y una terapia. Muy resumidamente, la ACT hunde sus raíces en el conductismo radical 
de Skinner que comparte el marco filosófico del contextualismo funcional. Este modelo terapéutico desarrolla su fundamentación conceptual gracias a la consolidación de la Teoría de los Marcos Relacionales (TMR) (28), que consiste en un análisis básico del lenguaje y la cognición humana bajo los principios del análisis de conducta. De este modo, desde la TMR el comportamiento simbólico o verbal se entiende como comportamiento relacional, es decir, un evento es verbal cuando adquiere sus funciones (significado) a través de su relación con otro. Otro aspecto importante de la TMR es el análisis del comportamiento de seguimiento de reglas. De este modo, los seres humanos podemos establecer relaciones entre eventos y adquirir nuevos comportamientos a través del seguimiento de reglas. Sin embargo, igual que las ventajas de ser verbales son inmensas, también el lenguaje se sitúa a la base del sufrimiento humano ya que se establecen y siguen relaciones que no son útiles o adecuadas y tienen consecuencias perjudiciales para la persona.

En este sentido, desde un punto de vista de la ACT, el sufrimiento psicológico es una parte inseparable de la existencia humana. Sin embargo y paradójicamente, vivimos en un contexto cultural en el que hay creencias muy extendidas de que hay que eliminar el sufrimiento. En este contexto, tratar de alterar o eliminar las sensaciones, los pensamientos, los sentimientos desagradables o angustiosos es algo considerado normal, adecuado, propio de personas cabales, aunque en ocasiones, las consecuencias de tales intentos sean muy negativas.

Un concepto clave es el de Evitación Experiencial (EE) (29-30) que se refiere a cuando una persona no está dispuesta a tener contacto con sus experiencias privadas y se comporta para alterar la forma o frecuencia de dichas experiencias y de los contextos que las ocasionan. De alguna manera, la reglas que están regulando este tipo de patrón son del tipo "para seguir adelante tengo que quitarme esta ansiedad". Cuando este patrón se establece de forma crónica se habla de Trastorno de Evitación Experiencial (TEE), que es un patrón inflexible, caracterizado por numerosos modos de respuesta que buscan evitar y suprimir o alterar la presencia de pensamientos, recuerdos, sensaciones y otros eventos privados, que resultan negativos o se acompañan de malestar, con el fin de poder vivir. Las formas en que una persona puede alterar o evitar los eventos privados son muy variadas, todas ellas producen un alivio inmediato al malestar, pero a largo plazo generan un sufrimiento mucho mayor.

\section{Características de la Terapia de Aceptación y Compromiso}

La ACT es un modelo de salud y no de enfermedad, ya que entiende que el sufrimiento es universal y su causa fundamental es la intromisión del lenguaje en áreas en las que no es funcional o no es útil. La ACT está especialmente indicada 
ORIGINALES Y REVISIONES

en aquellas personas en las que el TEE aparece de un modo crónico, y por tanto, constituye un patrón rígido e inflexible de interacción con el mundo privado.

El objetivo de la ACT es la aceptación de los eventos privados que están en el camino que el cliente elige para su vida. Lo realmente importante son los valores o direcciones que cada persona elige para su vida. Para ello, el paciente debe estar dispuesto a sentir o contemplar los eventos privados, a escoger y clarificar la dirección que quiere en su vida y dar pasos en esa dirección.

Es por ello que es importante identificar los contextos verbales que prevalecen en cada caso y que se identifican como trampas psicológicas generadas por el lenguaje. Hayes y Hayes (31) identificaron cuatro contextos verbales que pueden favorecer la aparición de trastornos psicológicos:

El contexto de literalidad a los contenidos cognitivos: se refiere al comportamiento de adherencia a los contenidos cognitivos (relaciones verbales derivadas), independientemente de las consecuencias que ello conlleve. El contenido mental rige el comportamiento y se torna insensible a las consecuencias de dicha literalidad sobre diferentes aspectos de la vida.

El contexto de la evaluación, comparación y categorización: dadas las características del lenguaje, una vez valoramos, comparamos o categorizamos un evento, nos relacionamos con dicho evento de forma indistinta a como lo hacemos con la valoración o comparación o características de la categoría que le hayamos atribuido. De esta forma, si la ansiedad es evaluada como algo malo, angustioso... nos relacionamos con la ansiedad en función de tales conceptos y, por tanto, será algo contra lo que luchar.

El contexto de la explicación o dar razones: la comunidad verbal ofrece explicaciones para la conducta de los individuos. Dichas explicaciones suelen ser explicaciones mentalistas, es decir, las conductas son provocadas por estados subjetivos subyacentes de la persona. Si tales estados son explicaciones o causas, el objetivo debería ser eliminar tales estados. Sin embargo, desde un punto de vista de $\mathrm{ACT}$ las razones no son causas.

El contexto del control emocional: como es frecuente que la comunidad piense que la conducta anormal es el resultado de eventos internos como emociones o pensamientos, se suele asumir que para que una persona sea capaz de controlar su conducta debe controlar los eventos internos que la causan (controla tu mente y controlarás tu vida).

\section{Componentes de la Terapia de Aceptación y Compromiso}

Los componentes de la ACT han sufrido variaciones en sucesivas versiones $(6,32)$, ya que se han ido incorporando los avances resultantes de la investigación 
básica y aplicada. Las actuaciones terapéuticas se dirigen, por un lado, a la clarificación de valores y el compromiso con la acción en el camino elegido, que conlleva la aceptación o el estar dispuesto a experimentar sin resistir los eventos privados que surjan en ese camino y, por otro lado, a la práctica de la de-fusión, es decir, a discriminar y tomar contacto o experimentar los pensamientos y sensaciones que sobrevienen, en el aquí y ahora, desde el yo como contexto al actuar con responsabilidad en la dirección elegida.

Los distintos componentes o fases de la ACT son los siguientes:

1. El establecimiento y mantenimiento de un contexto para la relación terapéutica

El terapeuta establecerá un contexto para la relación terapéutica en el que mostrará al paciente que lo importante en la sesión será su experiencia, minimizando la función del terapeuta como alguien que indica al paciente qué tipo de vida debe llevar, lo que debe sentir o pensar. De este modo, creará las condiciones necesarias para que el paciente experimente la contradicción entre lo que quiere conseguir y lo que realmente consigue. Al mismo tiempo, enfatizará que el paciente es una persona capaz de elegir el camino valioso para él y de afrontar el malestar. Para ello, utilizará metáforas y ejemplos con el objetivo de normalizar el sufrimiento y enseñar al paciente a aceptar sus eventos internos. Validar los sentimientos y experiencias del paciente y el propio compromiso con el paciente y con el proceso terapéutico son condiciones de un terapeuta ACT que deben estar presentes como contexto necesario para la terapia.

\section{Desesperanza creativa}

En esta fase se desarrollan actuaciones dirigidas a que el paciente experimente lo que desea, las actuaciones para conseguirlo y los resultados que obtiene a corto y largo plazo. De este modo, se facilita que el paciente se acerque a los modos en que ha tratado y trata de modificar los pensamientos, sentimientos, recuerdos, etcétera, que le resultan desagradables o angustiosos. Así puede considerar si los intentos por evitar sentirse mal han funcionado a largo plazo, es decir, cuál es el resultado. De esta forma, a través de metáforas el paciente toma contacto con la experiencia emocional derivada de que no tenemos la solución a sus problemas. Sin embargo, lo que no funciona, no funciona.

Con la desesperanza creativa se intenta que el paciente se haga consciente de la paradoja que supone intentar controlar los eventos privados, intentando eliminarlos o evitarlos, para poder vivir, pero, sin embargo, es algo que no le ha funcionado, sobre todo a largo plazo, lo que le produce insatisfacción en su vida. lución

3. La estrategia de control de los eventos privados es el problema, no la so-

Mediante paradojas, metáforas y ejercicios experienciales, el paciente aprende que el problema no lo constituyen los eventos privados en sí mismos, sino el 
ORIGINALES Y REVISIONES

afán de control de los mismos, y que una alternativa al control es la aceptación de dichos eventos.

\section{Clarificación de valores}

Es el contexto básico en ACT. Está presente desde el inicio de la terapia, ya que sin este contexto no existiría el sufrimiento ni ningún problema por resolver. Diferentes metáforas y ejercicios, como la metáfora del jardín, el funeral o el epitafio ayudan al paciente a clarificar sus valores y metas vitales. De esta forma, el paciente es capaz de identificar las trayectorias valiosas para su vida, los objetivos a corto y largo plazo, las acciones necesarias para conseguir estos objetivos y las barreras privadas que se interponen en la consecución de esos objetivos.

\section{Distanciamiento de los eventos privados}

Consiste en la observación de los eventos privados, desliteralizándolos y tomando perspectiva de los mismos. Para ello, se ayudará al paciente a diferenciar entre el acto de tener un pensamiento del pensamiento que se tiene y de la persona que lo tiene. La desliteralización tiene como objetivo debilitar la capacidad de control de los contenidos cognitivos. Así, el paciente aprenderá a ser consciente de si en un momento dado está actuando según lo que le indica su experiencia o no.

\section{Dimensiones del yo}

Con este componente se pretende que el paciente se dé cuenta de que puede tener un pensamiento y de que él es mucho más que eso. El yo es el contexto donde suceden todos los eventos privados, donde todos tienen cabida, ya que el yo es suficientemente grande como para tener todos los eventos cognitivos.

\section{El Trastorno Obsesivo-Compulsivo como Trastorno de Evitación Experiencial}

El TOC se puede conceptualizar como un claro ejemplo de Trastorno de Evitación Experiencial. En el TOC, la persona presenta experiencias internas (pensamientos, imágenes, estados emocionales, sensaciones) que le provocan acusado malestar. Debido al malestar que provocan en la persona, ésta rechaza estas experiencias internas, intentando suprimirlas o modificarlas mediante la puesta en marcha de diferentes compulsiones o rituales. La realización de compulsiones o rituales provoca un alivio a corto plazo del malestar, pero, sin embargo, compromete la vida de la persona a largo plazo, ya que cada vez los comportamientos neutralizadores conllevan más tiempo e interfieren más en la vida de la persona. Además, la compulsión queda reforzada por el alivio a corto plazo del malestar, por lo que la persona no llega a comprobar qué hubiera ocurrido si no la hubiera llevado a cabo. De esta forma, se crea un círculo vicioso en el que la persona siente malestar y realiza conductas con el fin de eliminar dicho malestar, lo que le impide comprometerse con los valores fundamentales de su vida. 
La ACT está especialmente indicada para personas que luchan contra sus eventos privados y no han obtenido ningún resultado, como es el caso del TOC, en el que la persona lucha contra sus obsesiones sin ser capaz de eliminarlas por completo. Esto hace que cada vez intente con más fuerza eliminar sus obsesiones, lo que termina provocando un estancamiento en su vida (32).

Los pacientes con TOC presentan una relación disfuncional con su experiencia privada, ya sean percepciones sensoriales, estados emocionales, imágenes o pensamientos. Predomina la fusión cognitiva, en la que el pensamiento es equivalente a al yo. Por lo tanto, para estos pacientes, el control de los eventos privados se convierte en algo fundamental, ya que no son capaces de distanciarse de dichos eventos. Por esto, el trabajo de desliteralización y de observación y toma de perspectiva de los eventos privados es muy importante. Así, se ayudará al paciente a diferenciar entre el acto de tener un pensamiento del pensamiento que se tiene y de la persona que lo tiene, aprendiendo a ser consciente de si en un momento dado está actuando fusionado con un pensamiento o una sensación (32).

En términos generales, los pacientes con TOC presentan un escaso dominio cognitivo del pasado y el futuro, con escaso autoconocimiento, ya que el momento presente se consume en las obsesiones. Se encuentran ligados a un Yo conceptual, ya que el Yo se subsume en las obsesiones sin tener conciencia del Yo como contexto. Por otro lado, al estar tan involucrados en la evitación persistente, estos pacientes se caracterizan por su inactividad. Existe escasa actividad dirigida a valores vitales y, sin embargo, una alta implicación en la realización de compulsiones. $\mathrm{Su}$ vida gira en torno al control de las obsesiones, sin establecer un compromiso con los valores de la persona (33).

Por tanto, se puede considerar al TOC como un TEE. En él están presentes todas las características que definen este tipo de trastorno, siendo la fundamental la necesidad de evitar y suprimir la presencia de pensamientos, recuerdos, imágenes y otros eventos privados que resultan negativos o se acompañan de malestar con el fin de poder vivir. Este modo de afrontamiento constituye un patrón inflexible de respuesta que resulta ineficaz y que provoca mayor malestar en la persona a largo plazo, comprometiendo la consecución de sus metas vitales.

La Terapia de Aceptación y Compromiso como tratamiento del Trastorno Obsesivo-Compulsivo

La ACT supone un cambio en el modo de enfocar el tratamiento con respecto a la EPR. La ACT no se centra en los síntomas (obsesiones y compulsiones), sino en la aceptación de experiencias internas y en el debilitamiento de la literalidad del 
ORIGINALES Y REVISIONES

lenguaje o fusión cognitiva. La ACT se enmarca de forma explícita en el contexto de los valores personales del paciente, al contrario de la EPR, que se utiliza de forma descontextualizada, lo que puede llevar a que el paciente utilice una estrategia de aproximación como una forma de evitar la ansiedad, con lo que se estaría potenciando la evitación del paciente y limitando aún más su vida (34).

El objetivo de la ACT es la aceptación de los eventos privados. Para ello, se desplaza el foco del tratamiento, de los síntomas a los valores y metas vitales del paciente. Éste es el medio para producir el cambio conductual. Para ello, es necesario que la persona se distancie de los contenidos psicológicos y desactive las funciones del lenguaje que favorecen la rigidez psicológica. La "defusión cognitiva" es uno de los procesos principales de la ACT. Este proceso implica organizar los contextos verbales a fin de disminuir la credibilidad de los pensamientos de la persona, así como disminuir la tendencia a responder en presencia de dichos pensamientos, mientras que no disminuye necesariamente su frecuencia ni se altera su forma (35).

Estas características sugieren que la ACT puede constituir una forma eficaz para el tratamiento del TOC, donde la aceptación y la defusión pueden resultar especialmente útiles. Las personas con TOC se centran de modo poco saludable en sus pensamientos obsesivos y realizan numerosos y variados comportamientos de evitación para alterar su forma o su frecuencia. En el tratamiento del TOC, la ACT persigue ayudar a la persona a crear una nueva relación con sus pensamientos obsesivos y sus sentimientos de ansiedad, en la que la obsesión pueda ser experimentada simplemente como otro pensamiento y la ansiedad sólo como una emoción más que se siente. Esto, a su vez, permitirá a la persona centrarse en hacer cosas que tienen sentido para su vida, en lugar de pasar grandes cantidades de tiempo tratando de disminuir las obsesiones o las sensaciones de ansiedad.

La ACT no es un conjunto particular de técnicas, sino que constituye un enfoque terapéutico que se dirige a seis procesos psicológicos: la aceptación, la defusión, el yo como contexto, ponerse en contacto con el momento actual, los valores y comprometerse con la acción, todo lo cual fomenta la flexibilidad conductual (8). Por tanto, se pueden llevar a cabo muchos procedimientos diferentes y ser todos ACT, siempre y cuando se trabaje sobre estos procesos. Así, la ACT no constituye una terapia protocolizada, sino que se trata de una aproximación más global consistente en una serie de procedimientos que el clínico va implementando a lo largo del desarrollo de la terapia, pudiéndose así adaptar a cada caso concreto.

Twohig (36) publica un trabajo en el que describe la aplicación de la ACT para un caso de TOC. Twohig señala que los seis procesos anteriormente mencionados raramente se realizan de forma secuencial, sino que más bien se van intercalando a lo largo de la terapia, de forma que el clínico decide en cada momento cuál es más útil. El proceso de aceptación implica permitir las experiencias internas sin 
llevar a cabo medidas para controlarlas. En el caso del TOC, el autor defiende que este proceso debe ser el primero a tratar en la terapia. En este proceso, el terapeuta trabaja con el paciente para ver si los intentos de solución que ha llevado a cabo hasta ahora le han resultado útiles, o si por el contrario, dichos intentos de solución han hecho que las obsesiones se vuelvan más frecuentes e intensas. El trabajo del terapeuta en esta fase es establecer las condiciones para que el paciente tenga la experiencia de que las obsesiones no pueden ser controladas a largo plazo, y que los intentos de controlar realmente le están haciendo más daño, es decir, que tratar de controlar el problema forma parte del problema. El terapeuta hace hincapié en este punto en que, debido a que estas estrategias han tenido resultados tan pobres, tratar de controlar las obsesiones puede ser parte del problema, y no parte de la solución. A pesar de que las obsesiones pueden disminuir al dejar de intentar controlarlas, la disminución de las obsesiones del paciente no es el foco de la ACT, ya que las obsesiones volverán a reaparecer ocasionalmente, y si el objetivo es la disminución de éstas el paciente seguirá en una continua lucha después del tratamiento.

El segundo proceso es la defusión cognitiva. Las personas con TOC suelen estar fusionadas cognitivamente, es decir, tienen poca conciencia de sus obsesiones como pensamientos sin significado. Por el contrario, suelen experimentar sus obsesiones como eventos literales y concretos. Las personas con TOC se asustan de sus pensamientos y de los efectos que éstos pueden tener. Disminuir esta fusión cognitiva supone que las obsesiones tendrían mucho menos control sobre la conducta de la persona. Para ello, Twohig (36), considera que debe crearse una relación terapéutica en la que las obsesiones sean tratadas simplemente como pensamientos y emociones, no como cosas reales. Según este autor, muchos de los procedimientos utilizados en otras terapias, como la discusión directa o el cuestionamiento socrático no se usarán en ACT. Estos procedimientos son incompatibles con ACT, ya que lo más probable es que se aumente la dependencia del paciente al pensamiento lógico y racional. En este punto, se pueden utilizar ejercicios como los anteriormente descritos ("metáfora de los pasajeros del autobús", repetir la obsesión o ejercicios de atención plena).

El tercer proceso es el Yo como contexto. Esto implica que hay un sentido inmutable de sí mismo que está siempre presente y no puede ser dañado. Sin el yo como contexto, las personas con TOC experimentarán dos procesos problemáticos: responderán a sus obsesiones como si estuvieran definidas por ellas y por lo tanto intentarán controlarlas, y responderán de manera coherente con sus pensamientos. El terapeuta trabajará para reforzar la presencia del yo como contexto y ayudará al paciente a ver la separación entre él y sus obsesiones. El objetivo es que el paciente experimente sus obsesiones como eventos que ocurren dentro de él, en lugar de como acontecimientos que le definen. Si el paciente detecta la separación entre sus experiencias internas y él mismo, estará en mejor disposición de no actuar de acuerdo con sus obsesiones. 
ORIGINALES Y REVISIONES

Otro de los procesos en ACT es ponerse en contacto con el momento presente, es decir, experimentar conscientemente los acontecimientos internos y externos que se están produciendo, sin evaluaciones o juicios. Estar en mayor contacto directo con lo que está ocurriendo en el momento presente va en contra de la fusión cognitiva, ya que elimina la evaluación verbal. Esto permite a las personas con TOC experimentar el mundo tal como es y no como está determinado por sus cogniciones.

Los valores se definen como cualidades de la vida que son perseguidos en todo momento, pero que nunca se pueden llegar a lograr completamente. Son las cosas que importan a la persona y por las que está dispuesta a luchar. En este punto, es importante el concepto de "elección", ya que deja de lado la fusión cognitiva. Tomar una decisión de seguir un valor es útil porque el paciente no tiene que tomar una decisión racional. Las sesiones sobre valores pueden ocurrir en cualquier punto en la terapia, pero comúnmente se producen al final, después del resto de procesos. El objetivo es hacer ver al paciente que una de las cosas que se interpone en el camino hacia la consecución de sus valores es la energía gastada en intentar controlar sus obsesiones. Que sus valores sean los que guíen sus acciones se presenta como una alternativa a que sea su experiencia interior la que guíe su comportamiento.

Por último, el proceso de comprometerse con la acción se centra en el cambio de comportamiento. El compromiso con la acción está ligado a los valores personales del paciente. Así, los ejercicios se realizan en función de los valores elegidos por el paciente, y nunca se hacen para controlar eventos privados. En segundo lugar, se anima al paciente a practicar los otros procesos de ACT mientras realiza los ejercicios. En tercer lugar, los ejercicios se estructuran por tiempo o actividad, no por la gravedad o el nivel de las obsesiones. Por último, es el paciente, no el terapeuta, el que elige el ejercicio.

En resumen, el objetivo de la ACT para el TOC es aumentar la aceptación de las obsesiones, notarlas como lo que son, y dirigirse hacia las direcciones de valor. En la mayoría de los casos, esto se traducirá en una reducción de la conducta compulsiva, pero sólo porque el comportamiento compulsivo generalmente interfiere con la búsqueda de los valores.

Uno de los estudios bien controlados que analiza los resultados de la aplicación de ACT en el TOC es el de Twohig, Hayes y Masuda (35), en el que aplican ACT a cuatro pacientes con TOC (dos con obsesiones de comprobación, uno de limpieza y otro de acumulación). El programa consistió en ocho sesiones de tratamiento, semanalmente, de una hora de duración. Los resultados muestran una reducción importante de las conductas compulsivas y los rituales durante el tratamiento, así como una retención de la mayor parte o de la totalidad de las ganancias durante el seguimiento, con niveles de compulsiones cercanos a cero en la fase de post tratamiento. También se observaron reducciones en los niveles de ansiedad 
y depresión durante el tratamiento, niveles que seguían disminuyendo a los tres meses de seguimiento. En todos los casos se observó un aumento de la aceptación de eventos internos y todos los pacientes calificaron el tratamiento como altamente aceptable. Los resultados se mantuvieron a los tres meses de seguimiento.

Este mismo protocolo de ocho sesiones fue comparado con entrenamiento en relajación muscular progresiva con 34 adultos con TOC (18 fueron tratados con ACT y 16 con relajación muscular progresiva) (37). En este estudio estuvieron representados todos los subtipos de TOC. Los resultados mostraron mejoras significativas en el grupo tratado con ACT, en comparación con el grupo tratado con relajación muscular progresiva, después del tratamiento y durante el seguimiento, mostrando también la ACT una mayor mejoría clínica. Los procesos de ACT produjeron cambios mayores y de forma más temprana en el TOC, en comparación con la relajación muscular progresiva. Este mismo estudio fue replicado por Twohig et al. (38) con una muestra de 79 pacientes con TOC.

Dehlin, Morrison y Twohig (39) aplicaron la ACT para el tratamiento de la escrupulosidad en cinco adultos con TOC. Obtuvieron buenos resultados, con una disminución en la frecuencia de las obsesiones y las actividades evitadas, disminución en medidas de depresión, mejor calidad de vida y mayor aceptación. Además, los pacientes calificaron la ACT como altamente aceptable y se lograron resultados favorables en un periodo muy corto de tiempo (12 horas de terapia por paciente). Por último, en los últimos años también en España se está haciendo un esfuerzo por integrar al mismo tiempo técnicas basadas en la aceptación con procedimientos de exposición en el tratamiento del TOC. Gutiérrez-Martínez, Luciano y Visdómine (40) exponen el tratamiento para un caso de TOC en el que utilizan técnicas basadas en la ACT al mismo tiempo que utilizan técnicas de exposición en el marco de la defusión cognitiva y para promover la aceptación de contenidos molestos, obteniendo resultados positivos al finalizar el tratamiento y manteniéndose éstos al año de seguimiento. Por su parte, Montero, Fernández y Pol (41) publican un estudio en el que utilizan la terapia cognitivo-conductual con componentes de aceptación y compromiso para el tratamiento de un caso de TOC, obteniendo una disminución significativa de la sintomatología obsesiva y compulsiva al finalizar el tratamiento y a los 6 meses de seguimiento, valorando además la paciente la terapia de manera positiva.

\section{Discusión.}

En el tratamiento del TOC, la ACT pretende ayudar a la persona a crear una nueva relación con sus pensamientos obsesivos y sus sentimientos de ansiedad. 
ORIGINALES Y REVISIONES

Más que controlarlos, se pretende que el paciente los acepte tal como lo que son, sólo pensamientos y sentimientos. La ACT contextualiza todo esto en el contexto de los valores y metas vitales del paciente, al contrario que la EPR, que se utiliza de un modo descontextualizado, lo que puede llevar al paciente a realizar las exposiciones como otra forma más de evitación de la ansiedad. El objetivo en ACT es ayudar a la persona a experimentar una obsesión por lo que es (es decir, un pensamiento) y que continúen haciendo lo que es importante para ellos (valores y metas). La presencia real de la obsesión no es el tema principal. Dicho de otra manera, el objetivo es ampliar el repertorio efectivo del individuo en presencia de eventos temidos (por ejemplo, la obsesión), lo que se ha denominado "flexibilidad psicológica".

Aunque, tanto EPR como ACT trabajan en definitiva la exposición, la EPR lo hace centrándose en los eventos temidos, como por ejemplo, contaminarse. La ACT, sin embargo, no lo hace centrándose en el evento temido concreto, sino que lo hace centrándose en el elemento funcional equivalente a todas las situaciones que están en el sustrato de las obsesiones y las compulsiones: la incertidumbre y el no control. Además de los factores explicativos mencionados anteriormente acerca de por qué en algunos pacientes la EPR no resulta eficaz (evitación cognitiva, falta de habituación a corto plazo, depresión, presencia de ideas sobrevaloradas), otro factor a considerar es la propia dinámica de la exposición. Muchas veces, las exposiciones a objetos o situaciones que se llevan a cabo en la EPR no funcionan porque, en realidad, el problema no se genera ni se mantiene por simples procesos de condicionamiento, sino que entra la influencia del lenguaje, que hace presente lo que no está presente y dota de valor o significado a cada situación descrita. Además, la mera exposición a objetos o situaciones de la obsesión no resulta eficaz para prevenir TOC más complejos, como ocurre en el caso del trastorno obsesivocompulsivo de la personalidad, que constituye un caldo de cultivo (un contexto psicológico) para el desarrollo de un TOC posterior.

Aunque existen hoy todavía pocos estudios que utilicen la ACT en el tratamiento del TOC, éste es un terreno actualmente en auge y los resultados preliminares disponibles hasta el momento apuntan a unos beneficios prometedores, derivados del cambio de enfoque terapéutico que proporciona. Aunque parece que la EPR puede resultar útil para algunos pacientes con TOC, para un importante porcentaje de pacientes, dada la naturaleza propiamente aversiva de la exposición a situaciones y objetos temidos, resulta un procedimiento altamente negativo, por lo que estos pacientes terminan abandonando la terapia antes de finalizarla. En este sentido, la ACT abre la puerta a un enfoque alternativo de los pensamientos y sentimientos aversivos, ayudando a la persona a crear una nueva relación con sus pensamientos obsesivos y sus sentimientos de ansiedad, persiguiendo la aceptación de los mismos. 


\section{BIBLIOGRAFÍA}

(1) Vallejo MA. Tratamientos psicológicos eficaces para el trastorno obsesivo compulsivo. Psicothema. 2001;13(3):419-427.

(2) Abramowitz JS, Taylor S, McKay D. Obsessive-compulsive disorder. Lancet. 2009;374:491-499.

(3) Fisher P, Wells A. Experimental modification of beliefs in obsessive-compulsive disorder: A test of the metacognitive model. Behav Res Ther. 2005;43:821-829.

(4) Foa EB, Liebowitz MR, Kozak MJ, Davies S, Campeas R, Franklin ME, et al. Randomized, placebocontrolled trial of exposure and ritual prevention, clomipramine, and their combination in the treatment of obsessive-compulsive disorder. Am J Psychiatry. 2005;162:151-161.

(5) McLean PD, Whittal ML, Thordarson DS, Taylor S, Söchting I, Koch WJ, et al. Cognitive versus behavior therapy in the group treatment of Obsessive-Compulsive disorder. J Consult Clin Psychol. 2001;69:205-214.

(6) Hayes SC, Strosahl KD, Wilson KG. Acceptance and Commitment Therapy. An experiential approach to behavior change. Nueva York: The Guilford Press; 1999.

(7) Hayes SC. A contextual approach to therapeutic change. In: Jacobson NS, editor. Psychotherapists in clinical practice. Cognitive and behavioral perspectives. Nueva York: The Guilford Press; 1987. p. 327-387.

(8) Hayes SC, Luoma JB, Bond FW, Masuda A, Lillis J. Acceptance and commitment therapy: model, process and outcomes. Behav Res Ther. 2006;44:1-25.

(9) Hayes SC, McCurry SM, Afari N, Wilson KG. Acceptance and Commitment Therapy (ACT). A therapy manual for the treatment of Emotional Avoidance. Reno, Nevada: Context Press; 1991.

(10) Carrascoso FJ, Valdivia S. Acceptance and Commitment Therapy (ACT) in the Treatment of Panic Disorder: Some Considerations from the Research on Basic Processes. Rev Int Psicol Ter Psicol. 2009;9(3):299-315.

(11) Eifert GH, Forsyth JP, Arch J, Espejo E, Keller M, Langer D. Acceptance and Commitment Therapy for Anxiety Disorders: Three Case Studies Exemplifying a Unified Treatment Protocol. Cogn Behav Pract. 2009;16:368-385.

(12) Roemer L, Orsillo SM, Salters-Pedneault K. Efficacy of an acceptance-based behavior therapy for generalized anxiety disorder: evaluation in a randomized controlled trial. J Consult Clin Psychol. 2008;76(6):1083-1089.

(13) Wetherell JL, Afari N, Ayers CR, Stoddard JA, Ruberg J, Sorrell JT, et al. Acceptance and Commitment Therapy for generalized anxiety disorder in older adults: a preliminary report. Behav Ther. 2011;42(1):127-134.

(14) Bach P, Hayes SC. The use of acceptance and commitment therapy to prevent the rehospitalization of psychotic patients: A randomized controlled trial. J Consult Clin Psychol. 2002;70:1129-1139.

(15) García-Montes JM, Pérez-Álvarez, M. Fundamentación experimental y primeras aplicaciones clínicas de la terapia de aceptación y compromiso en el campo de los síntomas psicóticos. Rev Latinoam Psicol. 2005;37(2):379-393.

(16) Luciano MC, Gómez S, Hernández M, Cabello F. Alcoholismo, evitación experiencial y Terapia de Aceptación y Compromiso (ACT). Análisis y Modificación de Conducta. 2001;27(113):333-371. 
ORIGINALES Y REVISIONES

(17) Velasco JA, Quiroga E. Formulación y solución de un caso de abuso de alcohol en términos de aceptación y compromiso. Psicothema. 2001;13:50-56.

(18) Luciano MC, Cabello F. Trastorno de duelo y Terapia de Aceptación y Compromiso (ACT). Análisis y Modificación de Conducta. 2001;27:399-424.

(19) Markanday S, Data-Franco J, Dyson L, Murrant S, Arbuckle C, McGillvray J, et al. Acceptance and commitment therapy for treatment-resistant depression. Aust N Z J Psychiatry. 2012;46(12):1198-1199.

(20) Walser RD, Karlin BE, Trockel M, Mazina B, Barr Taylor C. Training in and implementation of Acceptance and Commitment Therapy for depression in the Veterans Health Administration: therapist and patient outcomes. Behav Res Ther. 2013;51(9):555-563.

(21) McCracken LM, Sato A, Taylor GJ. A trial of a brief group-based form of acceptance and commitment therapy (ACT) for chronic pain in general practice: pilot outcome and process results. J Pain. 2013;14(11):1398-1406.

(22) McCracken LM, Sato A, Wainwright D, House W, Taylor GJ. A feasibility study of brief group-based acceptance and commitment therapy for chronic pain in general practice: recruitment, attendance, and patient views. Prim Health Care Res Dev. 2013;19:1-12.

(23) Williams MT, Mugno B, Franklin M, Faber S. Symptom dimensions in obsessivecompulsive disorder: phenomenology and treatment outcomes with exposure and ritual prevention. Psychopathology. 2013;46(6):365-376.

(24) Bolton D, Perrin S. Evaluation of exposure with response-prevention for obsessive compulsive disorder in childhood and adolescence. J Behav Ther Exp Psychiatry. 2008;39(1):11-22.

(25) Williams MT, Crozier M, Powers M. Treatment of Sexual-Orientation Obsessions in Obsessive-Compulsive Disorder Using Exposureand Ritual Prevention. Clin Case Stud. 2011;10(1):53-66.

(26) Foa EB, Simpson HB, Liebowitz MR, Powers MB, Rosenfield D, Cahill SP, et al. Six-month follow-up of a randomized controlled trial augmenting serotonin reuptake inhibitor treatment with exposure and ritual prevention for obsessive-compulsive disorder. J Clin Psychiatry. 2013;74(5):464-469.

(27) Whittal ML, Robichaud M, Thordarson DS, McLean PD. Group and individual treatment of obsessive-compulsive disorder using cognitive therapy andexposure plus response prevention: a 2-year follow-up of two randomized trials. J Consult Clin Psychol. 2008;76(6):1003-1014.

(28) Hayes SC, Barnes-Holmes D, Roche B. Relational frame theory: A post-Skinnerian account of human language and cognition. New York: Plenum Press; 2001.

(29) Bach P, Moran D. ACT in practice: Case conceptualization in Acceptance and Commitment Therapy. Oakland, CA: New Harbinger; 2008.

(30) Hayes SC, Gifford EV. The trouble with language: Experiential avoidance, rules, and the nature of verbal events. Psychological Science. 1997;8:170-173.

(31) Hayes SC, Hayes LJ. Understanding verbal relations. Reno, NV: Context Press; 1992.

(32) Wilson KG, Luciano MC. Terapia de Aceptación y Compromiso (ACT): Un tratamiento conductual orientado a los valores. Madrid: Ediciones Pirámide; 2002.

(33) Twohig MP, Moran DJ, Hayes SC. A functional contextual account of obsessive compulsive disorder. In: Woods DW, Kantor J, editors. A modern behavioral analysis of clinical problems. Reno, NV: Context Press; 2007. p. 117-156.

(34) Gutiérrez O, Luciano C, Visdómine C. ACT y TOC: integrando las técnicas basadas en la aceptación y en la exposición con el estudio de un caso. En: Páez M, Gutiérrez O, editores. Múl- 
tiples aplicaciones de la Terapia de Aceptación y Compromiso (ACT). Madrid: Pirámide; 2012. p. 89-112.

(35) Twohig MP, Hayes SC, Masuda A. Increasing Willingness to Experience Obsessions: Acceptance and Commitment Therapy as a Treatment for Obsessive-Compulsive Disorder. Behav Ther. 2006;37:3-13.

(36) Twohig MP. The Application of Acceptance and Commitment Therapy to ObsessiveCompulsive Disorder. Cogn Behav Pract. 2009;16:18-28.

(37) Twohig MP. A randomized clinical trial of acceptance and commitment therapy versus progressive muscle relaxation in the treatment of obsessive compulsive disorder. Diss Abstr Int: Section B: The Sciences and Engineering. 2007;68(7-B):4850.

(38) Twohig MP, Hayes SC, Plumb JC, Pruitt LD, Collins AB, Hazlett-Stevens H, et al. A randomized clinical trial of acceptance and commitment therapy vs. progressive relaxation training for obsessive compulsive disorder. J Consult Clin Psychol. 2010;78:705-716.

(39) Dehlin JP, Morrison KL, Twohig MP. Acceptance and Commitment Therapy as a Treatment for Scrupulosity in Obsessive Compulsive Disorder. Behav Modif. 2013;37(3):409-430.

(40) Gutiérrez-Martínez O, Luciano C, Visdómine C. ACT y TOC: integrando las técnicas basadas en la aceptación y en la exposición con el estudio de un caso. En: Páez M, Gutiérrez-Martínez O. Múltiples aplicaciones de la Terapia de Aceptación y Compromiso (ACT). Madrid: Ediciones Pirámide; 2012. p. 89-112.

(41) Montero c, Fernández LC, Pol J. Terapia cognitivo-conductual con componentes de aceptación y compromiso en un caso de trastorno obsesivo-compulsivo. Rev Asoc Esp Neuropsiq. 2013;33(117):123-134. 